\title{
Thiol-disulphide homeostasis in patients with surgical site infections
}

\author{
Duygu Mert ${ }^{1}$, Murat Alışık ${ }^{2}$, Cihat Oğan ${ }^{1}$, Salim Neşelioğlư ${ }^{3}$, Mustafa Ertek ${ }^{1}$, Özcan Erel ${ }^{3}$ \\ ${ }^{1}$ University of Health Sciences Dr. Abdurrahman Yurtaslan Ankara Oncology Training and Research Hospital, \\ Department of Infectious Diseases and Clinical Microbiology, Ankara, Turkey \\ ${ }^{2}$ Mus State Hospital, Department of Medical Biochemistry, Mus, Turkey \\ ${ }^{3}$ Ankara City Hospital, Department of Medical Biochemistry, Ankara, Turkey
}

\begin{abstract}
Objectives: In infectious diseases, various inflammatory cells are active and reactive oxygen species are produced to fight against intra cellular and extra cellular microorganisms. This leads to an increase in potential antioxidant capacity and free radical production. Thiol is an important antioxidant. Thiols enter oxidation reactions with oxidative molecules and it form disulphide bonds. The aim of this study was to evaluate the dynamic thiol/disulphide homeostasis in patients with surgical site infection (SSI).
\end{abstract}

Methods: Sixty-eight patients with SSI and 66 healthy persons (control group) were included in this study. Complete blood count, biochemistry, erythrocyte sedimentation rate, C-reactive protein values and thiol levels were studied in blood of the patients at 0th day and 10th day. The blood thiol disulphide homeostasis was analysed using a new automated method developed by Erel and Neselioglu.

Results: Native thiol, total thiol, albumin levels and native thiol/total thiol ratio were found significantly lower in the blood samples at day 0th compared to 10th day. There was a statistically significant difference between the patient group and the control group in IMA, native thiol, total thiol, albumin, disulphide levels, disulphide/native thiol, disulphide/total thiol and native thiol/total thiol ratios

Conclusions: The thiol-disulphide balance is impaired in SSI. The elevated disulphide/total thiol ratio and disulphide/native thiol ratio, and reduced native thiol/total thiol ratio indicate increased oxidation in SSI. There is also a strong association between CRP, ESH and thiol-disulphide parameters. Thiol-disulphide homeostasis may potentially be of benefit in inflammatory response. J Microbiol Infect Dis 2020; 10(3):160-166.

Keywords: Surgical site infections, thiol-disulphide homeostasis, inflammatory response

\section{INTRODUCTION}

Surgical site infections (SSI) cause serious morbidity and mortality. It remains one of the most important problems of the surgeon.

SSI are infections of the incision or organ or space that occur after surgery. SSI, operation is infection that occurs within $30-90$ days following [1].

In infectious diseases, various inflammatory cells are active and reactive oxygen species are produced to fight against intra cellular and extra cellular microorganisms [2]. This leads to an increase in free radical production and potential antioxidant capacity [3]. Thiols are compounds that contain sulfur and important antioxidants [4]. Thiols enter oxidation reactions with oxidative molecules and it form disulphide bonds. Dynamic thiol/disulphide homeostasis is required for detoxification, regulation of signaling pathways, regulation of apoptosis and enzymatic reactions $[5,6]$.

In cases such as ischemia, acidosis, free radical damage, the amino terminal binding region in albumin is replaced and the binding capacity for metals is reduced. This different form of the albumin is ischemia modified albumin (IMA) [7]. The mechanism of IMA is thought that the effect

Correspondence: Dr. Duygu Mert, University of Health Sciences Dr. Abdurrahman Yurtaslan Ankara Oncology Training and Research Hospital, Infectious Diseases and Clinic Microbiology Clinic, Ankara, Turkey

E-mail: drduygumert@hotmail.com

Received: 25 May 2020 Accepted: 23 August 2020

Copyright (C JMID / Journal of Microbiology and Infectious Diseases 2020, All rights reserved 
of reactive oxygen species is a consequence of change in the metal binding region of albumin [8].

The aim of this study was to evaluate the dynamic thiol/disulphide homeostasis in patients with SSI and to determine if thiol-sulphide values are important parameters following inflammation.

\section{METHODS}

This study was a prospective observational trial. Two groups consisting of patients with SSI diagnosis and healthy persons in a tertiary hospital were included between January 1, 2015 and March 1, 2016 in this study.

Sixty-eight patients (37 females, 31 males) with SSI according to the criteria established by the Centers for Disease Control and Prevention (CDC) were included and 66 healthy persons (29 females, 37 males) were included as control group.

SSI are infections of the incision or organ or space that occur after surgery within 30-90 days following [1] Patients with SSI were questioned about discharge, hyperemia and edema in their wounds and accompanying chronic diseases [1].

The control group was consisted healthy persons who underwent normal physical examination and routine laboratory tests.

The venous blood samples from patients with SSI were taken at 0th day before antibiotic treatment and at 10th day during treatment. The blood was taken from the control group. The blood was put into biochemistry tubes.

At the same time, complete blood count, biochemistry, erythrocyte sedimentation rate (ESR) and C-reactive protein (CRP) values were studied in blood of the patients at 0th day and $10^{\text {th }}$ day.

The blood was centrifuged for 5 minutes at 2000 $\mathrm{rpm}$ in a centrifuge. The serum from upper part of the blood was obtained. The serum samples were put into Eppendorf tubes and stored at $80^{\circ} \mathrm{C}$ in deep freezing until biochemical analysis was performed. The blood thiol disulphide homeostasis was analyzed using a new automated method developed by Erel and Neselioglu [9].
For each participant, the SSI-related parameters and demographic data were recorded on a standard form designed by the researchers.

The study protocol was approved by the ethics committee (Yildirim Beyazit University Faculty of Medicine Clinical Research Ethics Committee (Approval date: June 10, 2015 and number: 146). Written informed consent was obtained from all persons.

\section{Biochemical analysis}

In this study, modified Ellman reagent was used to determine total thiol levels in blood samples. The classical Ellman reagent was modified by adding formaldehyde solution [9]. In a study by Erel et al., the modified Ellman reagent was used to measure the plasma thiol level [9]. The significant correlation was found between modified and original methods [9].

The main principle of Erel and Neselioglu method is the reduction of disulphide bonds to reactive thiol groups in the presence of $\mathrm{NaBH} 4$ [9]. The formaldehyde reaction neutralises the unreacted $\mathrm{NaBH} 4$ and it prevents further reduction of Ellman's reagent (5,5'-dithiobis-(2nitrobenzoic acid) or DTNB) [9).

The serum thiol-disulphide profile tests were performed using a new automated method with an automated analyzer (Cobas c501, Roche, Mannheim, Germany). The modified Ellman reagent was used to measure the total thiol content in the samples. The half of difference between total and native thiol concentrations were value of disulphide bonds. The disulphide/total thiol, disulphide/native thiol and native thiol/total thiol ratios were also calculated using the reaction results.

\section{Additional measurements}

The complete blood count, biochemistry, ESR and CRP values were also studied at 0th day before antibiotic treatment and at 10th day during treatment in patients with SSI.

\section{Statistical analysis}

Statistical analyzes were made using the package program called SPSS (IBM SPSS Statistics 20). Frequency tables and descriptive statistics were used to interpret the findings. 
Parametric methods were used for measurement values suitable for normal distribution. Non-parametric methods were used for measurement values that were not suitable for normal distribution. In accordance with the parametric methods, "Paired Sample t" test ( $t-$ table value) statistics was used to compare two dependent groups with normal distribution, in accordance with the non-parametric methods, "Wilcoxon" test (Z-table value) method was used to compare two dependent groups with the measured values.

"Independent Sample t" test (t-table value) statistics were used to compare two independent groups with normal distribution. "Mann-Whitney U" test (Z-table value) statistics were used to compare two independent groups without normal distribution.

"x2-cross" tables were used to examine the relationship between two qualitative variables. $P$ value $<0.05$ was considered statistically significant.

\section{RESULTS}

Sixty-eight patients [37 females (54.4\%) and 31 males (45.6\%)] with SSI were included and 66 healthy persons [29 females (43.9\%) and 37 males $(56.1 \%)]$ were included in this study.

The mean age of the patients was $59.13 \pm 16.54$ years and the mean age of the control group was $55.48 \pm 14.49$ years. There was no statistically significant difference between mean age of the control group and mean age of the patient group (Table 1).

Patients with SSI had a follow-up period of 10 days. Venous blood was collected from the patients at the 0th day before treatment and at the 10th day of treatment. Patients with septic shock were excluded from this study.

There was a significant difference in hemoglobin, hematocrit, CRP, ESR, native thiol, total thiol and albumin levels, disulphide/native thiol, disulphide/total thiol and native thiol/total thiol ratios in the blood samples at 0th and 10th day. CRP and ESR were significantly higher in the blood samples at 0th day compared to 10th day $(p=0.012$ and $p=0.005)$. Native thiol, total thiol and albumin levels were significantly lower in the blood samples at 0th day compared to 10th day $(p=0.001, p=0.032$ and $p=0.000$ respectively). However, disulphide/native thiol and disulphide/total thiol ratios were significantly higher in the blood samples at 0th day compared to 10th day $(p=0.001$ and $p=0.004)$. Native thiol/total thiol ratio was significantly lower at day 0th compared to 10th day $(p=0.004)$ (Table 2).

On the other hand, there was no statistically significant difference between erythrocyte middle cell volume, white blood cell count, IMA and disulphide levels between 0th day and 10th day (Table 2).

There was a significant difference in thiol levels between patients with SSI and healthy controls. There was a statistically significant difference between the patient group and the control group in IMA, native thiol, total thiol, albumin, disulphide levels, disulphide/native thiol, disulphide/total thiol and native thiol/total thiol ratios (Table 3 ).

There was a significant difference between the patient group and the control group in native thiol levels. The native thiol levels were lower in the patient group than in the control group (patient:102.7 [3.7-287.5] $\mu \mathrm{mol} / \mathrm{L}$, control:350.3 [127.1-527.4] $\mu \mathrm{mol} / \mathrm{L}, \mathrm{z}=-9.626, \mathrm{p}=0.000$ ) (Table 3).

Table 1. Basic characteristics of patient and control group.

\begin{tabular}{cccc}
\hline $\begin{array}{c}\text { Basic } \\
\text { features } \\
(\mathrm{N}=134)\end{array}$ & SSI $(\mathrm{n}=68)$ & $\begin{array}{c}\text { Control } \\
(\mathrm{n}=66)\end{array}$ & $\begin{array}{c}\text { Statistical } \\
\text { analysis } \\
\text { Possibility }\end{array}$ \\
\hline Age (year) & $59.13 \pm 16.54$ & $55.48 \pm 14.49$ & $\begin{array}{c}\mathrm{t}=1.356 \\
\mathrm{p}=0.177 \\
\mathrm{X}^{2}=1.930, \\
\mathrm{p}=0.165\end{array}$ \\
\hline Gender (F) & $37(54.4 \%)$ & $29(43.9 \%)$ \\
\hline
\end{tabular}

SSI: Surgical site infection.

Total thiol and albumin levels were statistically lower in the patient group than in the control group ( $p=0.000$ and $p=0.000$ ) (Table 3$)$. IMA and disulphide levels were significantly higher in the patient group than in the control group $(p=0,000$ and $p=0,000$ ) (Table 3).

The native thiol/total thiol ratio was significantly higher in the control group $(p=0.000)$, while the disulphide/native thiol and disulphide/total thiol ratios were significantly higher in the patient group ( $p=0.000$ and $p=0.000$ ) (Table 3 ). 
Table 2. Laboratory values of patients with SSI at Oth and 10th day in plasma samples.

\begin{tabular}{|c|c|c|c|}
\hline $\mathrm{SSI}^{1}$ & $0^{\text {th }}$ day & $10^{\text {th }}$ day & $\begin{array}{l}\text { Statistical analysis, Possibility } \\
\qquad\left(\mathrm{p}^{2}\right)\end{array}$ \\
\hline Hemoglobin (11-16 g/dL) & $10.2[7.1-15.2]$ & $11.2[6.9-14.6]$ & $Z=-2.696, p=0.007$ \\
\hline Hematocrit (37-54\%) & $32.5[22.6-48.2]$ & $35.2[21.2-44.8]$ & $Z=-2.383 p=0.017$ \\
\hline $\begin{array}{l}\text { MCV (erythrocyte middle cell volume) (80-100 } \\
f L)\end{array}$ & $87.7[70.1-110.2]$ & 86.9 [73.4-96.7] & $Z=-0.756 p=0.450$ \\
\hline White blood cell count $\left(4-10 \times 10^{3}\right.$ cells $\left./ \mu \mathrm{L}\right)$ & $8.2[1.4-34.3]$ & $8.4[2.3-24.8]$ & $Z=-0.690 p=0.490$ \\
\hline CRP $(0-5 \mathrm{mg} / \mathrm{L})$ & $82.5[2.4-309.0]$ & $61.8[3.4-265.1]$ & $Z=-2.515 p=0.012$ \\
\hline Erythrocyte sedimentation rate (ESR) $(\mathrm{mm} / \mathrm{h})$ & $67.6[6.0-123.0]$ & $64.1[11.0-142.0]$ & $Z=-2.786 p=0.005$ \\
\hline IMA (ischemia modified albumin) (U/ml) & $76.0[46.5-82.3]$ & $75.6[65.1-81.7]$ & $Z=-0.631 p=0.528$ \\
\hline Native thiol $(\mu \mathrm{mol} / \mathrm{L})$ & $102.7[3.7-287.5]$ & $135.8[7.9-289.6]$ & $Z=-3.422 p=0.001$ \\
\hline Total thiol, $(\mu \mathrm{mol} / \mathrm{L})$ & $\begin{array}{l}174.8[39.6- \\
392.8]\end{array}$ & $\begin{array}{l}204.5[27.0- \\
\quad 358.4]]\end{array}$ & $Z=-2.139 p=0.032$ \\
\hline Albumin $(3.5-5.2 \mathrm{~g} / \mathrm{dL})$ & $4.75 \pm 0.88$ & $5.16 \pm 0.90$ & $t=-3.974 p=0.000$ \\
\hline Disulphide, $(\mu \mathrm{mol} / \mathrm{L})$ & $38.1[5.5-72.5]$ & $33.5[9.6-83.4]$ & $Z=-1.143 p=0.253$ \\
\hline Disulphide/native thiol ${ }^{\star}, \%$ & $41.9[5.3-1478.1]$ & $25.3[5.2-1029.7]$ & $Z=-3.315 p=0.001$ \\
\hline Disulphide/total thiol* ${ }^{\star} \%$ & $22.6[4.8-48.4]$ & $16.8[4.8-47.7]$ & $Z=-2.878 p=0.004$ \\
\hline Native thiol/total thiol ${ }^{*}, \%$ & 54.9 [3.3-90.4] & $66.4[4.6-90.5]$ & $Z=-2.878 p=0.004$ \\
\hline
\end{tabular}

\section{DISCUSSION}

Thiols are susceptible to oxidation and thiols can react with disulfides through thiol disulfide exchange reactions. As these disulfide bonds are reversible, they can be reduced to thiols depending upon the organism's oxidantantioxidant balance [9]. The thiol-disulphide balance is an important member of various processes such as immunoreaction, apoptosis, antioxidant defense and regulation of enzyme activity [10]. Impaired thiol-disulphide balance have been shown for example, in a variety of diseases such as myocardial infarction, diabetes mellitus, preeclampsia and cancer [11].

Thiol-disulphide homeostasis has also been evaluated in various infectious diseases including Crimean-Congo hemorrhagic fever (CCHF), acute tonsillopharyngitis and acute brucellosis [12-14]. In all three studies, the thioldisulphide balance was found to be impaired [12-14].

In a study by Tufan et al., a positive or negative relationship was found between thiol disulphide tests with some oxidant and antioxidant parameters in CCHF. In patients with CCHF increased disulphide/native thiol and disulphide/total thiol ratios, decreased total antioxidant status, and increased total oxidant status were found [12]. 
In a study by Kara et al., there was a negative correlation between CRP values and the number of white cells with native and total thiol levels in acute tonsillopharyngitis. Patients with viral and bacterial tonsillopharyngitis had lower native thiol levels compared to healthy children $(p<0.001$ and $p=0.008$, respectively). Total thiol levels were lower in both groups compared to healthy children $(p=0.002$ for viral, $p=0.011$ for bacterial). The ratio of native thiol/total thiol in each patient group was found to be lower than the control group $(p<0.001$ for viral, $p=0.017$ for bacterial). Disulphide/native thiol and disulphide/total thiol ratios were significantly higher in viral $(p<0.001$ in both) and bacterial tonsillopharyngitis patients $(p=0.017$ in both) than healthy children [13].

Table 3. The difference in plasma thiol levels between patients with SSI and control group.

\begin{tabular}{lccc}
\hline Variable $(\mathrm{n}=134)$ & $\mathrm{SSI}(\mathrm{n}=68)$ & Control $(\mathrm{n}=66)$ & $\begin{array}{c}\text { Statistical analysis* } \\
\text { Possibility }\left(\mathrm{p}^{1}\right)\end{array}$ \\
\hline IMA (ischemia modified & $76.0[46.5-82.3]$ & $73.9[57.0-78.4]$ & $\mathrm{Z}=-5.132 \mathrm{p}=0.000$ \\
albumin $(\mathrm{U} / \mathrm{mL})$ & $102.7[3.7-287.5]$ & $350.3[127.1-527.4]$ & $\mathrm{Z}=-9.626 \mathrm{p}=0.000$ \\
Native thiol $(\mu \mathrm{mol} / \mathrm{L})$ & $174.8[39.6-392.8]$ & $392.9[194.7-547.5]$ & $\mathrm{Z}=-9.166 \mathrm{p}=0.000$ \\
Total thiol $(\mu \mathrm{mol} / \mathrm{L})$ & $4.8[2.4-6.9]$ & $6.4[3.2-7.0]$ & $\mathrm{Z}=-8.630 \mathrm{p}=0.000$ \\
Albumin $(3.5-5.2 \mathrm{~g} / \mathrm{dL})$ & $38.1[5.5-72.5]$ & $18.4[2.1-67.3]$ & $\mathrm{Z}=-5.581 \mathrm{p}=0.000$ \\
Disulphide, $(\mu \mathrm{mol} / \mathrm{L})$ & $41.9[5.3-1478.1]$ & $5.1[0.4-29.7]$ & $\mathrm{Z}=-9.141 \mathrm{p}=0.000$ \\
Disulphide/native thiol ${ }^{*}, \%$ & $22.16 \pm 9.47$ & $5.45 \pm 3.77$ & $\mathrm{t}=13.351 \mathrm{p}=0.000$ \\
Disulphide/total thiol ${ }^{*}, \%$ & $55.68 \pm 18.93$ & $89.10 \pm 7.54$ & $\mathrm{t}=-13.350 \mathrm{p}=0.000$ \\
Native thiol/total thiol ${ }^{*}, \%$ & & &
\end{tabular}

In a study by Kolgelier et al. acute brucellosis has been shown to impair balance between thiol-disulphide pairs. In patients with acute brucellosis, disulphide/native thiol ratios and disulphide/total thiol ratios were significantly higher and native thiol/total thiol ratios were significantly lower $(p<0.001$, rates for all) than in the healthy controls [14].

In a study by Ozyazici et al., the native thiol and total thiol levels, native thiol/total thiol ratio were found to be statistically significantly lower in patients with acute appendicitis than in the control group $(p<0.001)$. Disulphide level, disulphide/native thiol and disulphide/total thiol ratios were found higher in the acute appendicitis group than in the control group $(p<0.001)$. There was a positive correlation between CRP and disulphide/native thiol and disulphide/total thiol ratios in the acute appendicitis group [15].

SSI are infections that occur after surgery. In this study was evaluated the thiol-disulphide balance in SSI.

In this study, CRP, ESR levels, disulphide/native thiol and disulphide/total thiol ratios were significantly higher in the blood samples at 0 th day compared to 10th day $(p=0.012, p=0.005$, $p=0.001$ ve $p=0.004$, respectively).

Hemoglobin, hematocrit, native thiol, total thiol, albumin levels and native thiol/total thiol ratio were significantly lower in the blood samples at 0th day compared to 10th day $(p=0.007$, $\mathrm{p}=0.017, \quad \mathrm{p}=0.001, \quad \mathrm{p}=0.032, \quad \mathrm{p}=0.000$ and $\mathrm{p}=0.004$ respectively) (Table 3 ).

At Oth day, native thiol: 102.7 [3.7-287.5] $\mu \mathrm{mol} / \mathrm{L}$, total thiol: 174.8 [39.6-392.8] $\mu \mathrm{mol} / \mathrm{L}$, albumin levels: $4.75 \pm 0.88 \mathrm{gr} / \mathrm{dL}$, disulphide/native thiol: 41.9\% [5.3-1478.1] and disulphide/total thiol: 22.6\% [4.8-48.4]. At 10th day, native thiol: 135.8 [7.9-289.6] $\mu \mathrm{mol} / \mathrm{L}$, total thiol: 204.5 [27.0-358.4] $\mu \mathrm{mol} / \mathrm{L}$, albumin levels: $5.16 \pm 0.90 \mathrm{gr} / \mathrm{dL}$, disulphide/native thiol: $25.3 \%$ [5.2-1029.7] and disulphide/total thiol: $16.8 \%$ [4.8-47.7].

Native thiol, total thiol, albumin levels and native thiol/total thiol ratio were found to be significantly lower in the patient group than in the control group ( $\mathrm{p}=0.000, \mathrm{p}=0.000, \mathrm{p}=0.000$ ve $\mathrm{p}=0.000$ ) (Table 4). In addition, disulphide levels, disulphide/native thiol and disulphide/total thiol ratios were significantly higher in the patient group than in the control group $(p=0.000$, $p=0.000$ ve $p=0.000$ ) (Table 4). 
IMA is an indicator in the evaluation of ischemic events [16]. Serum IMA levels increase in patients with cardiac ischemia, liver cirrhosis, pulmonary embolism, end-stage renal failure, cerebrovascular diseases, cancer, systemic sclerosis, intrauterine disorders, diabetes mellitus, multiple trauma and policystic ovarian disease [17-24].

In the present study, IMA level was significantly higher in the patient group than in the control group $(p=0.000)$ (Table 4$)$. This was thought to be due to wound ischemia associated with SSI.

These results showed that the thiol-disulphide balance was impaired in patients with SSI. There was a significant difference in thiol levels between patients with SSI and healthy controls.

Bacterial infections produce oxidizing molecules such as myeloperoxidase and NADPH oxidase in granulocytes with the aid of oxidative enzymes. In this way, as thiol levels drop, disulphide levels increase [19]. In the present study, native thiol and total thiol levels were found to be low in the patient group.

\section{Conclusion}

As a result, the thiol-disulphide balance is impaired in SSI. In pre-treatment depleted native thiol and total thiol levels has been associated with decreasing effects against oxidative stress and inflammation. The elevated disulphide/total thiol ratio and disulphide/native thiol ratio, and reduced native thiol/total thiol ratio indicate increased oxidation in SSI. There is also a strong association between CRP, ESH and thioldisulphide parameters. CRP and ESH are positive acute phase reactant. For this reason, thiol-disulphide homeostasis may potentially be of benefit in inflammatory response. Therefore, thiol-disulphide homeostasis may provide new approaches for SSI's follow-up.

Limitations of the study: These parameters among patients with prosthetics and nonprosthetics or response to treatment and nonresponse to treatment have not been studied.

\section{ACKNOWLEDGMENTS}

Acknowledgments: We would like to thank Melih Uzunoglu for his support in statistical analyses.

Funding: There is no financial support for this study.
Declaration of Conflicting Interests: The authors declare that they have no conflict of interest.

\section{REFERENCES}

1.National Healthcare Safety Network, Centers for Disease Control and Prevention. Surgical site infection (SSI) event. http://www.cdc.gov/nhsn/pdfs/pscmanual/9pscssicurre nt.pdf. Published January 2020. Accessed January 01, 2020.

2. Esen R, Aslan M, Kucukoglu ME, et al. Serum paraoxonase activity, total thiols levels, and oxidative status in patients with acute brucellosis. Wien Klin Wochenschr 2015;127(11-12):427-433.

3. Dundaroz R, Erenberk U, Turel O, Demir AD, Ozkaya E, Erel O. Oxidative and antioxidative status of children with acute bronchiolitis. J Pediatr (Rio J) 2013;89(4):407-411.

4. Cadenas E. Biochemistry of oxygen toxicity. Annu Rev Biochem 1989; 58:79-110.

5. Kuo LM, Kuo CY, Lin CY, Hung MF, Shen JJ, Hwang TL. Intra cellular glutathione depletion by oridonin leads to apoptosis in hepatic stellate cells. Molecules 2014; 19(3):3327-3344.

6. Prabhu A, Sarcar B, Kahali S, et al. Cysteine catabolism: A novel metabolic pathway contributing to glioblastoma growth. Cancer Res. 2014; 74(3):787796.

7. Roy D, Quiles J, Gaze D, Collinson P, Kaski J, Baxter GF. Role of reactive oxygen species on the formation of the novel diagnostic marker ischaemia modified albumin. Heart 2006; 92(1): 113-114.

8. Gidenne S, Ceppa F, Fontan E, Perrier F, Burnat P. Analytical performance of the Albumin Cobalt Binding (ACB) test on the Cobas MIRA Plus analyzer. Clin Chem Lab Med 2004; 42(4):455-461.

9. Erel O, Neselioglu S. A novel and automated assay for thiol/disulphide homeostasis. Clin Biochem 2014; 47(18):326-332.

10. Valko M, Leibfritz D, Moncol J, Cronin MT, Mazur $\mathrm{M}$, Telser J. Free radicals and antioxidants in normal physiological functions and human disease. Int $\mathrm{J}$ Biochem Cell Biol 2007; 39:44-84.

11. Kundi $H$, Ates I, Kiziltunc $E$, et al. A novel oxidative stress marker in acute myocardial infarction; thiol/disulfide homeostasis. Am J Emerg Med 2015; 33:1567-1571.

12. Tufan ZK, Hasanoglu I, Kolgelier S, et al. A retrospective controlled study of thiol disulfide homeostasis as a novel marker in Crimean Congo hemorrhagic fever. Redox Rep 2016; 21:1-5.

13. Kara SS, Erel O, Demirdag TB, Yayla BCC, Gulhan B, Neselioglu S. Alteration of thiol-disulfide 
homeostasis in acute tonsillopharyngitis. Redox Rep 2016; 20:1-5.

14. Kolgelier S, Ergin M, Demir LF, et al. Impaired thiol-disulfide balance in acute brucellosis. Jpn $\mathrm{J}$ Infect Dis 2017;70:258-262.

15. Ozyazici S, Karateke F, Turan U, et al. Novel Oxidative Stress Mediator in Acute Appendicitis: Thiol/Disulphide Homeostasis. Mediators Inflamm 2016; 2016:6761050.

16. Bar-Or D, Lau E, Winkler J. A novel assay for cobalt-albumin binding and its potential as a marker for myocardial ischemia- a preliminary report. J Emerg Med 2000;19(4): 311-315.

17. Cakir M, Karahan S, Mentese A, et al. IschemiaModified Albumin Levels in Children with Chronic Liver Disease. Gut Liver 2012; 6(1): 92-97.

18. Turedi S, Patan T, Gunduz A, et al. Ischemiamodified albumin in the diagnosis of pulmonary embolism: an experimental study. Am J Emerg Med 2009; 27(6):635-640.

19. Turedi S, Cinar O, Yavuz I, et al. Differences in ischemia modified albumin levels between end stage renal disease patients and the normal population. $\mathrm{J}$ Nephrol 2010; 23(3):335-340.

20. Gunduz A, Turedi S, Mentese A, et al. Ischemiamodified albumin levels in cerebrovascular accidents. Am J Emerg Med 2008; 26(8):874-878.

21. Ma S, Xu W, Wei C. Role of ischemia-modified albumin and total homocysteine in estimating symptomatic lacunar infarction in type 2 diabetic patients. Clin Biochem 2011; 44(16):1299-1303.

22. Shao-gang $M$, Yao J, Wen $H$, Feng $B$, Wen $X$, Wei-nan Y. Evaluation of Ischemia Modified Albumin and C-Reactive Protein in Type 2 Diabetics with and without Ketosis. Biomark Insights 2012; 7:19-26.

23. Can M, Demirtas S, Rolat O, Yıldız A. Evaluation of effects of ischemia on the albumin cobalt binding (ACB) assay in patients exposed to trauma. Am J Emerg Med 2006; 23(7):537-539.

24. Caglar G, Oztas E, Karadag D, Pabuccu R, Demirtas S. Ischemia Modified Albumin and cardiovascular risk markers in polycystic ovary syndrome with or without insulin resistance. Fertil Steril 2011; 95(1): 310-313. 